\title{
CIENCIA ANORMAL. EL ESCENARIO COSMOLÓGICO CON MATERIA OSCURA
}

\author{
Alan Heiblum Robles*
}

¿Qué debemos pensar del sonado escenario cosmológico con materia oscura? ¿Se trata de un escenario revolucionario, en crisis, o el resultado de la ciencia normal? En el presente trabajo se argumenta que para describir al escenario favorito de la cosmología actual hace falta un concepto de una ciencia erigida sobre sus propias anomalías.

Palabras clave: cosmología, materia oscura, revolución, crisis, ciencia normal.

\section{ABNORMAL SCIENCE. THE COSMOLOGICAL STAGE WITH DARK MATTER}

What should we think of the famous cosmological scenario regarding dark matter? Is it about a revolutionary scenario or the result of normal science? The present paper argues that to describe current cosmology's favorite scenario, it takes a concept based on a science build up on its own anomalies.

Keywords: cosmology, dark matter, revolution, crisis, normal science.

* Universidad Nacional de Tres de Febrero (UNTREF), Buenos Aires, Argentina. Correo electrónico: mulbieh@gmail.com 


\section{El escenario cosmológico con materia oscura}

EL CENSO UNIVERSAL, REALIZADO POR LA MISIÓN PLANCK EN 2013 , confirma un extraño resultado: la materia ordinaria (polvo de estrellas) representa apenas el $15.5 \%$ de la materia del universo, mientras que el restante $84.5 \%$ lo conforma una entidad aún no detectada ni plenamente identificada que llamamos materia oscura.

La materia oscura, una hipótesis primero dejada de lado, pero ahora aplaudida por el grueso de la comunidad científica, conlleva una historia larga y llena de matices. Para efectos del presente trabajo basta con un breve recordatorio, en tres tiempos, de su constitución. El primero de ellos es en 1933 cuando Zwicky advirtió que para que la mecánica clásica describiera correctamente los clusters de galaxias era necesario suponer que estos contienen mucha más materia que solo la visible ${ }^{2}$. En un segundo momento, entre los años setenta y ochenta, los modelos de formación galáctica sugirieron que las galaxias espirales no serían estables, a menos que estuvieran sumidas en un halo de materia no visible ${ }^{3}$. Simultáneamente, un exiguo trabajo astronómico determinó -contra toda expectativa- que las curvas de rotación de la galaxias son planas (las galaxias rotan como si, de hecho, tuvieran mucha más materia que solo la visible) ${ }^{4}$. En un tercer momento, llegados los años noventa, las simulaciones de estructuras cósmicas descartaron que esta nueva entidad interactuara con el campo electromagnético y pudiera estar conformada por materia ordinaria y relativista ${ }^{5}$. Así, según el escenario favorito de la actualidad, la existencia de materia oscura fría, conformada por partículas no ordinarias aún no identificadas, es un hecho, no obstante, aún no se cuenta con una detección en toda regla. ¿Qué debemos pensar de este sonado escenario cosmológico?, ¿se trata de una nueva conquista de la ciencia o de un novedoso atolladero?

1 Planck Mission 2013, Planck Legacy Archive. 21/03/2013, Planck 2013 results, XVI, Cosmological parameters, arXiv: 1303.5076v1 [astro-ph.CO].

2 Cfr. Zwicky, F., "Der Rotverschiebung von extragalaktischen Neblen", Act. Helv. Phys., 6, 1933, pp. 110-127.

3 Cfr. Ostriker, J.P. \& Peebles, P.J.E., A numerical study of flattened galaxies: or cancold galaxies survive, Astrophys. J., 186, 1973, pp. 467-480.

4 Cfr. Rubin, V., Ford, K. \& Thonnard, N., "Rotational properties of $21 \mathrm{Sc}$ galaxies with a large range of luminosities and RADII, from NGC $4605(\mathrm{r}=4 \mathrm{kpc})$ to UGC $2885(\mathrm{r}=122 \mathrm{kpc})$ ", The Astronomical Journal, 238, June 1. 1980, pp.471-487.

5 Cfr. Navarro, J.F., Frenk, C.S. \& White, S.D.M., "The diversity and similarity of simulated cold dark matter haloes", Mon. Not. R. Astron. Soc., 402, 2010, pp. 21-34. 
Aunque virtualmente nadie en el campo de los estudios de la ciencia acepta el modelo de Kuhn en La estructura como correcto en detalle", durante el 2010 fueron publicados dos diagnósticos antagónicos en clave del Kuhn de La estructura. En el primero de ellos, Einasto, físico conocido por su contribución a los perfiles de los halos oscuros, afirma que el escenario con materia oscura constituye una nueva revolución científica ${ }^{7}$. Einasto no aporta mayores argumentos, sino que basa su afirmación en la lectura que Tremaine diera en el simposio sobre materia oscura de la Unión Astronómica Internacional (IAU) en 1985, en Princeton. Dicho trabajo fue publicado en 1987, lo cual resulta interesante pues se trata del mismo año de la publicación de Theocharis y Psimopoulos en Nature, donde Kuhn es declarado - junto a Popper, Lakatos, y Feyerabend- un enemigo de la ciencia ${ }^{8}$. En cambio, Tremaine lo sitúa como amigo de la ciencia.

\section{La materia oscura: ¿una revolución?}

Un resumen del texto de Tremaine en sus propias palabras es el siguiente:

Creo que desde hace varias décadas, la astronomía extragaláctica ha sido ciencia normal. El paradigma correspondiente es dificil de describir por completo pero incluiría la suposición de que las leyes de Newton son correctas, que la masa de una galaxia está contenida mayoritariamente en sus estrellas visibles, que la función de masa inicial es más o menos una ley de potencia, y más o menos lo mismo en todas partes, que la formación de galaxias no es sesgada, etc. (...) El primer signo de la crisis, es que alguna anomalía parece ser algo más que un puzzle de la ciencia normal (desde mi punto de vista, esto ocurrió alrededor de 1974, con la curva de rotación del M31 de Roberts-Whithurst y el artículo de Ostriker, Peebles y Yahil proponiendo halos masivos) (...) De acuerdo a Kuhn, el intervalo de tiempo entre el viejo paradigma y la enunciación del nuevo es siempre de las dos últimas décadas, si contamos el comienzo de la crisis a partir de 1974, entonces tenemos menos de diez años de espera ${ }^{9}$.

Este es el diagnóstico de 1987, aprobado por Einasto en 2010, ignorando medio siglo de epistemología. Mucho trabajo se ha hecho antes y después de 1962, incluido el del propio Kuhn, y si lo que se busca es caracterizar una revolución científica, simplemente no puede dejarse de lado.

6 Nickles, Tн., "Scientific Revolutions", en: Zalta, E.N. (Ed.), The Stanford Encyclopedia of Philosophy, (Winter 2013 Edition), forthcoming URL $=<$ http://plato.stanford.edu/archives/win2013/entries/scientific-revolutions/>. Todas las traducciones son mías.

7 Einasto, J., "Dark Matter", Astronomy and Astrophysics, in: Systems Encyclopedia of Life Support (EOLSS), Oxford, arXiv: 0901.0632 v2 [astro-ph.CO], 2010.

8 Theocharis, T. \& Psimopoulos, M., "Where science has gone wrong", Nature, Vol. 329, No. 6140, 15 October 1987, pp. 595-598.

9 Tremaine, S., 1987, A Historical Perspective on Dark Matter, in: Kormendy, J. \& Knapp, G.R. (Eds.), IAU Symposium, Vol. 117, Dark matter in the universe, p. 547. 
Ahora bien, para el primer Kuhn, el indicador de una revolución es la incomensurabilidad, donde el mapa de su argumento es bien conocido: los científicos comparten un paradigma y realizan ciencia normal rodeados por anomalías. Algunas de éstas se vuelven inmanejables y llevan a una crisis. La crisis se resuelve con un cambio de paradigma y el establecimiento de una nueva ciencia normal. Los científicos antes y después de una revolución trabajan en mundos distintos ${ }^{10}$.

El concepto de inconmensurabilidad -en un principio compartido y luego abandonado por Feyerabend-, presenta una montaña de problemas ${ }^{11}$, pero puede entenderse como la interrelación de tres aspectos: "cambios en los problemas y estándares que definen la disciplina, cambios en los conceptos usados para establecer y solucionar esos problemas, y cambio de mundo"12.

Por mi parte, sostengo que durante el desarrollo histórico del escenario cosmológico con materia oscura, estrictamente, no hay incomensurabilidad. Desde el punto de vista teórico que es el que Kuhn aborda, tomando 1917 -año del primer modelo cosmológico realizado por Einstein- como inicio del período a estudiar y finalizando con los resultados de la misión Planck 2013, no se tiene incomensurabilidad, sino una medida común en varios e importantes aspectos. Los cosmólogos de entonces, como los de ahora, hablan el mismo idioma y articulan la misma estructura teórica. Ésta última es la de la relatividad general con la gravitación de Newton como límite clásico. En este marco se han postulado una serie de modelos, que claramente son susceptibles de discernimiento por sus ventajas y desventajas, al tiempo que utilizan los mismos recursos teóricos (e.g. la constante cosmológica) e idénticas hipótesis de trabajo (e.g. el principio cosmológico).

En breve, no hay un cambio mayor en la definición de la disciplina. En 1970 -antes de que surgiera la crisis mencionada por Tremaine-, el reconocido discípulo de Hubble, Allan Sandage, sentenció que la cosmología era la búsqueda de dos números $\mathrm{H}_{0}$ y W (la constante de Hubble, que da cuenta de la velocidad de expansión del universo, y la densidad de materia, que da cuenta de la geometría del universo), descripción que, consensuadamente, aún ajusta ${ }^{13}$.

Tampoco hay un cambio mayor en el establecimiento y la solución de problemas. Por ejemplo, en su trabajo de 1937, Zwicky recomienda usar el efecto relativista de las lentes gravitacionales en un contexto de materia oscura para determinar con precisión la canti-

10 Cfr. Kunn, Tн. S., La Estructura de las Revoluciones Cientificas, F.C.E., Buenos Aires, 2004.

11 Cfr. Lewowicz, L., Del relativisimo lingüistico al relativismo ontológico en el último Kuhn, D.P. Universidad de la República, Montevideo, 2005.

12 Oberheim, E. \& Hoyningen-Huene, P., “The Incommensurability of Scientific Theories”, en: Zalta, E.N. (Ed.), The Stanford Encyclopedia of Philosophy (Spring 2013 Edition), URL $=<$ http://plato.stanford.edu/archives/ spr2013/entries/incommensurability/>.

13 Cfr. Coles, P., Cosmology. A very short introduction, Oxford University Press, Oxford, 2001. 
dad de materia de las galaxias ${ }^{14}$. Es decir, Zwicky sugería utilizar la práctica que hoy día es usual.

Por último, el léxico no ha cambiado más que en otras disciplinas. La mayoría del instrumental ha sido actualizado, pero, en principio, sigue siendo usado de la misma manera. La mayoría de los viejos astrónomos están al día, ejerciendo su profesión competentemente. A grandes rasgos, la disciplina, su práctica, sus objetos y sus productos son tan inefables $\mathrm{u}$ ostensibles como antes.

Todo esto significa que nuestros cosmólogos del siglo XXI trabajan en el mismo mundo teórico que sus colegas del siglo XX. Es decir, en completa oposición a lo que sugirieron Einasto y Tremaine, no hay razones de peso que nos hagan siquiera cavilar de que se trata de una revolución alla Kuhn. Pero esto no es sólo culpa del recuento parcial que hicieran; tampoco hay mucho que nos permita pensar que las descripciones de Kuhn en su trabajo de 1962 ajusten del todo. A primera vista, una noción central como la de ciencia normal no parece satisfactoria para el escenario cosmológico. Pues no ocurrió, como según Kuhn sí era esperable, que el escenario se desarrollara junto a ciertas anomalías (ni siquiera fue el caso que los modelos hayan sido construidos en medio de un océano de anomalías, como pretendía Lakatos). Lo que en verdad ocurrió es que el escenario con materia oscura se construyó sobre las anomalías mismas. Sus propias siglas ( $\Lambda \mathrm{CDM}$ : $\Lambda$ de energía oscura y CDM de cold dark matter) son las de las entidades hipotéticas invocadas para dar cuenta de los desacuerdos entre teoría y observaciones. Esto significa que el escenario transformó sus anomalías en sus postulados. Siguiendo este tren de pensamiento se puede afirmar que el principal escenario cosmológico de la actualidad estaría funcionando como ciencia anormal, si bien no-revolucionaria.

Si nos abstenemos de nociones como incomensurabilidad y ciencia normal y escudriñamos la cosmología buscando no innovaciones teóricas, sino innovaciones experimentales y conceptuales, entonces la cosmología luce como un fascinante ejemplo de una disciplina en perpetua metamorfosis. El instrumental ha adquirido un poder portentoso y cada uno de los componentes del escenario cosmológico $\Lambda \mathrm{CDM}$ muestra una impresionante deriva conceptual. El universo dinámico de las ecuaciones de la relatividad, al principio no fue reconocido, y medio siglo después cristalizó en el modelo del Big Bang. La constante cosmológica, primero un "error" y luego un "acierto", pasó de ser un ingrediente para modelar un universo estático a modelar uno con expansión acelerada. La materia oscura, que empezó como un caso aislado de materia ordinaria presuntamente faltante en clusters y luego en galaxias, pasó de pensarse primero como caliente y luego como fría, y ahora se le adscribe un rol en la formación y en la estabilidad de estructuras, entendiéndola además como un componente mayoritario del universo.

14 Zwicky, F., “On masses of nebulae and clusters of nebulae”, Astrophys. J., 86, 1937, pp. 217-246, pp. 237-238. 
Todo lo contrario, para el primer Kuhn, la ciencia no-revolucionaria no debía producir novedad: "la característica más sorprendente de los problemas de investigación normal que acabamos de ver es quizá la de cuán poco aspiran a producir novedades importantes, conceptuales o fenomenales" ${ }^{\prime 15}$. Entonces, queda claro que el Kuhn de La estructura se pierde esta constante novedad en la ciencia no-revolucionaria porque, aunado a que prefiere la dimensión terminológica a la conceptual, sobrevalora su dimensión teórica, al tiempo que desestima sus dimensiones instrumentales y experimentales ${ }^{16}$.

\section{La materia oscura: ¿una crisis?}

Ahora bien, si a la fecha el escenario con materia oscura no constituye una revolución, pero tampoco es "ciencia normal" en términos kuhnianos, aún quedaría pensarlo como un escenario en crisis. Un diagnóstico así existe y resulta muy llamativo que sea del mismo año 2010, en que aparece su diagnóstico antitético. Revisemos la afirmación del físico Robert Sanders, bien conocido por sus contribuciones a la gravedad modificada ${ }^{17}$, de que el escenario cosmológico actual estaría en crisis:

Personalmente, creo que hay una crisis... ¿Hasta qué punto los experimentadores detendrán la búsqueda de estas partículas elusivas y pasarán a actividades con mayor probabilidad de producir resultados positivos? ¿Hasta qué punto los teóricos van a cansarse de más y más conjeturas especulativas sobre la naturaleza de hipotética materia indetectable ${ }^{18}$.

Este diagnóstico es compartido por Pavel Kroupa, que junto con sus colegas, clama ya desde el título de su artículo -también del 2010- que es hora de moverse "hacia un nuevo paradigma de la formación de estructuras"19. La argumentación de Kroupa puede ser parafraseada de la siguiente manera: es difícil encontrar una prueba que decida entre el escenario con materia oscura y los alternativos, porque todos responden al mismo tipo de problemas. Sin embargo, no es imposible. El escenario con materia oscura presenta incoherencias al nivel de las galaxias, donde la gravedad modificada funciona bien. Una posible solución para estas incoherencias se consigue al invocar una nueva fuerza oscura, que medie entre la materia ordinaria y la materia oscura. Opciones de este tipo llevan a los adherentes del escenario con materia oscura a decir que, con el tiempo, los problemas a nivel galáctico serán resueltos. Pero estas medidas ad hoc implican rehuir al menos cinco

15 Kunn, Th. S., La Estructura de las Revoluciones Cientificas, op. cit., p. 68.

16 Cfr. Hacking, I., "Introductory Essay" to The Structure of Scientific Revolution by Thomas S. Kuhn, 50th Anniversary Edition, The University of Chicago Press, Chicago, 2012. p. xvii.

17 Escenario cosmológico alternativo al poblado por materia oscura. Vid.: MiLgrom, M., "A modification of the Newtonian dynamics as a possible alternative to the hidden mass hypothesis", Astrophys. J., 270, 1983, pp. 365370.

18 Sanders, R.H., The Dark Matter Problem. A historical perspective, Cambridge University Press, Cambridge, pp. 169-170, 2010.

19 Kroupa, P. et al., "Local-Group tests of dark-matter concordance cosmology. Towards a new paradigm for structure formation?", Astron. Astrophys., 523, A32, [arXiv:1006.1647 [astro-ph.CO], 2010. 
problemas, que parecen ser irreconciliables si se suponen galaxias dominadas por materia oscura, pero que son sencillamente resueltos con una teoría de gravedad modificada.

Ahora bien, ¿se trata de una crisis tipo Kuhn? El término crisis está utilizado para señalar la difícil situación de aquel que defiende lo indefendible, pero advertir si también se utiliza en el sentido de Kuhn no es inmediato. Que en los escritos aparezca explícitamente el término "paradigma" no nos debe llevar a ninguna conclusión apresurada. El vocablo "paradigma" se volvió viral, y la comunidad astrofísica, como tantas otras, lo usa de forma trivial y trivializante. De la misma manera "cambio de paradigma" se ha repetido ad nauseam de forma acrítica e irreflexiva, tornándose simples palabras huecas que dicen poco o nada, pero esconden innumerables confusiones semánticas.

En su libro de 2010, Sanders definitivamente sí utiliza el calificativo "crisis" en el sentido del primer Kuhn. Pero, nuevamente, no parece que la caracterización del primer Kuhn se preste para trabajar la crisis que presenta el escenario con materia oscura, y esto por la siguiente razón. Las crisis en el recuento del primer Kuhn, se gestan, crecen y se resuelven, presentando un contraste con la ciencia normal. Pero, una inspección histórica de la hipótesis de la materia oscura muestra que el contraste entre crisis y ciencia normal es nulo, como se muestra en el hecho de que la hipótesis de la materia oscura es tan vieja como la relatividad general misma. De manera que, si se acepta el diagnóstico de crisis para el caso de la hipótesis de la materia oscura, se tendría una crisis permanente o en continua gestación, y estos no son conceptos kuhnianos. Ahora bien, una crisis permanente o en continua gestación sí parecería capturar las líneas del desarrollo histórico de la cosmología moderna para el caso de la materia oscura.

\section{Lo "terrible"}

A continuación, pasaré revista a un caso histórico que guarda una profunda relación con la idea de materia oscura:

He hecho una cosa terrible, he postulado una partícula que no puede ser detectada ${ }^{20}$.

Esta frase pertenece a Wolfgang Pauli y su trasfondo es el siguiente: en 1930, para salvar la aparente no conservación de energía-momento del decaimiento beta, Pauli postuló como "un remedio desesperado" -según sus propias palabras- la existencia de una partícula que llamó "neutrón" (hoy neutrino) $)^{21}$, sin carga eléctrica, con spin de 1/2 y una masa no mayor que 0.01 veces la masa del protón.

20 Cit. en: Sutton, Ch., Spaceship Neutrino, Cambridge University Press, Cambridge, 1992, p. xi.

21 En 1932, Chadwick identificó las partículas masivas y neutras que hoy día llamamos neutrones y la partícula de Pauli fue rebautizada en 1934 por Fermi utilizando el diminutivo italiano. 
Inseguro e incómodo con la autoría de una hipótesis no medible con los instrumentos entonces disponibles, Pauli no se sentía suficientemente seguro para publicar nada hasta no tener algún indicio de por dónde buscar una detección experimental. Sin embargo, pronto esta medida ad hoc terminó obteniendo resultados afortunados. Como afirma Sutton: "dos desarrollos cambiaron dramáticamente la situación: el descubrimiento de los procesos de fisión y el desarrollo de los gigantes detectores líquidos luminiscentes" ${ }^{22}$. Nótese que, a diferencia de la materia oscura, la comunidad científica no celebró confiadamente la existencia del neutrino antes de obtener su detección. Para 1956, dieciséis años después de su postulación, el neutrino ya había sido detectado ${ }^{23}$. En los años posteriores, además, fue identificada en tres tipos. Hoy sabemos que los neutrinos son un componente esencial para entender el entramado universal. La Misión Planck también confirmó que su masa no es la predicha por Pauli, pero que tampoco es nula, sino que tiene un límite superior de masa de $0.23 \mathrm{eV}$.

Pauli calificó de terrible la postulación de una entidad prima facie no detectable, y lo hizo con razón. Me parece importante explicitar qué es lo que la hace terrible, para ello primero analizaré aquello que no la hace ser terrible. Las condiciones experimentales cambian constantemente, de manera que lo que es indetectable hoy, puede ser trivialmente detectable mañana. De esta manera, descartando lo absolutamente indetectable -si es que dicho concepto tiene sentido-, la indetectabilidad en sí misma es peligrosa, pero no terrible. Cuando uno postula una entidad ad hoc para resolver un desencuentro entre principios matemáticos y evidencia, uno se embarca en una línea de investigación que, por necesidad, debe ser improbable, ya que se está hablando de algo que nunca antes fue visto. Pero buscar lo improbable tampoco es terrible. Ahora bien, la apertura debida a la novedad improbable de la entidad da paso a que la hipótesis pueda ser remodelada cada vez que las experiencias den un resultado negativo en su detección. Es decir, se trata de una hipótesis altamente infalseable, cuestión que, si bien es indeseable, tampoco es terrible, al menos mientras uno no se adscriba a un criterio de demarcación popperiano donde lo que no es falseable es no científico. Por supuesto, declinar dicho criterio tampoco es terrible.

Si en lo anterior no estoy errado, el calificativo de terrible se tendría que aplicar por una razón diferente a las vertidas. Mi explicación es la siguiente: la situación es terrible porque hace del proyecto opuesto, a saber, el reconocimiento de la violación de un principio matemático y el consiguiente requerimiento de revisar con circunspección la coherencia del corpus involucrado, un proyecto únicamente opcional y siempre postergable. En otras palabras, la estrategia desesperada, consistente en convertir los desajustes empíricos de los principios matemáticos en hipótesis sobre la existencia de entidades aparentemente indetectables, esto es, centra la práctica científica en las anomalías mismas. Una ciencia

22 Sutton, Ch., Spaceship Neutrino, op. cit., p. xi.

23 Cowan, C.L. Jr., Reines, F., Harrison, F.B., Kruse, H.W., Mcguire, A.D., "Detection of the Free Neutrino: a Confirmation", Science, 124 (3212), 1956, pp. 103-104. 
que ya no se dedique a resolver las anomalías, sino a absorberlas, puede fácilmente tornarse en un edificio apologético, insondablemente abstracto e insípido, que se autoproclama victorioso aún sin pruebas. Esto es lo terrible.

De lo aquí vertido se sigue que la materia oscura es entonces una hipótesis terrible, pues constituye un nuevo paso en la estrategia consistente en dar credibilidad a una entidad difícilmente detectable, en lugar de revisar el aparato matemático que la invoca. Una ciencia con estas características es una ciencia que se abalanza en la dirección de una ampliación y sofisticación de la abstracción de sus matemáticas sin la necesidad de un avance simultaneo en la claridad de las interpretaciones de sus formalismos, ni en la elucidación de sus fundamentos. De proseguir esta dirección, se corre el riesgo de que la ciencia matemática termine hecha un ovillo, sorda e hipnotizada por su propio despliegue técnico, escapando de rama en rama en abstracciones, cada vez más lejos de sus raíces concretas. Una prestidigitación de cálculo y formalismos que en una pesadillesca compulsión a la repetición se ahoga en la especulación sin fin, en lugar de respirar en la gracia de la prueba.

\section{Conclusiones}

En el presente artículo contrapuse dos diagnósticos realizados por los propios artífices de los escenarios cosmológicos en pugna. Para el primero de ellos, el escenario con materia oscura es una suerte de revolución de la ciencia. El segundo, en cambio, afirma que la materia oscura está lejos de dar un panorama satisfactorio y completo de la imagen astronómica actual del universo. Para esta segunda opinión, la cosmología contemporánea no atravesaría una revolución, sino una crisis. Ahora bien, una nueva imagen se descubre si soltamos el concepto de ciencia normal y consideramos el concepto de ciencia anormal para aquella ciencia que se desarrolla en sus propias anomalías.

El concepto de ciencia normal excluye el concepto de crisis. La ciencia anormal lo puede incluir. Si esta nueva descripción resultara buena, el escenario cosmológico actual con materia oscura, en tanto ciencia anormal, estaría en crisis, pero esta crisis no se debería a que se sostiene la vigencia de un escenario que no resuelve con total coherencia la totalidad de la evidencia cosmológica -es decir, no se debería a la quiebra de una matriz disciplinar que preludia una nueva ciencia normal-, sino a que la comunidad científica estaría operando como si fuera un hecho lo que hasta el momento solo es una singular entidad hipotética que aún no consigue el correlato empírico adecuado. En otras palabras, el principal problema -lo más terrible- del escenario cosmológico con materia oscura es que no se auto-reconoce como terrible.*

*Artículo recibido: 21 de noviembre de 2014. Aceptado: 15 de diciembre de 2014. 


\section{Bibliografía}

Cowan, C.L. Jr., Reines, F., Harrison, F.B., Kruse, H. W., Mcguire, A.D., "Detection of the Free Neutrino: a Confirmation”, Science, 124 (3212), 1956, pp. 103-104.

EInASto, JAAn, "Dark Matter", Astronomy and Astrophysics, in Encyclopedia of Life Support Systems (EOLSS), Oxford, U.K., arXiv: 0901.0632 v2 [astro-ph.CO], 2010.

Kroupa, P. Eт Al., "Local-Group tests of dark-matter concordance cosmology. Towards a new paradigm for structure formation?", Astron. Astrophys., 523, A32, [arXiv:1006.1647 [astro-ph.CO], 2010.

Navarro, J.F., Frenk, C.S. \&White, S.D.M, “The diversity and similarity of simulated cold dark matter haloes”, Mon. Not. R. Astron. Soc., 402, 2010, pp. 21-34.

Nickles, Thomas, "Scientific Revolutions", Zalta, Edward N. (Ed.), The Stanford Encyclopedia of Philosophy (Winter 2013 Edition), forthcoming URL $=<$ http://plato.stanford. edu/archives/win2013/entries/scientific-revolutions/>.

Oberheim, Eric \& Hoyningen-Huene, Paul, "The Incommensurability of Scientific Theories", Zalta, Edward N. (ed.), The Stanford Encyclopedia of Philosophy (Spring 2013 Edition), URL $=<$ http://plato.stanford.edu/archives/spr2013/entries/incommensurability/>.

Ostriker, J.P. \& Peebles, P.J.E., "A numerical study of flattened galaxies: or cancold galaxies survive”, Astrophys. J., 186, 1973, pp. 467-480.

Planck Mission 2013, Planck Legacy Archive, 21/03/2013, Planck 2013 results, XVI, "Cosmological parameters", arXiv: 1303.5076v1 [astro-ph.CO].

Rubin, V., Ford, K. \& Thonnard, N., "Rotational properties of 21 Sc galaxies with a large range of luminosities and RADII, from NGC 4605 (r=4kpc) to UGC 2885 ( $\mathrm{r}=122 \mathrm{kpc}$ )", The Astronomical Journal, 238, June 1 1980, pp. 471-487.

SAnders, Robert H., The Dark Matter Problem. A historical perspective, Cambridge University Press, Cambridge, 2010.

Sutton, Christine, Spaceship Neutrino, Cambridge University Press, Cambridge, 1992.

Theocharis, T. \& Psimopoulos, M., "Where science has gone wrong”, Nature, Vol. 329, No. 6140, 15 October 1987, pp. 595-598. 
Tremaine, S., “A Historical Perspective on Dark Matter", in Kormendy, J. \& Knapp, G.R. (Eds.), IAU Symposium, Vol. 117, Dark matter in the universe, 1987.

Zwicky, Fritz, "Der Rotverschiebung von extragalaktischen Neblen", Act. Helv. Phys., 6, 1933, pp. 110-127.

----------------, “On masses of nebulae and clusters of nebulae”, Astrophys. J., 86, 1937, pp. 217-246, pp. 237-238. 